\title{
Silencing of MEF2D by siRNA Loaded Selenium Nanoparticles for Ovarian Cancer Therapy
}

This article was published in the following Dove Press journal:

International Journal of Nanomedicine

\author{
Changbing Wang, ${ }^{1,2, *}$ Yu Xia, ${ }^{1,3, *}$ \\ Shaochuan Huo, ${ }^{4,5} *$ Diwen Shou, ${ }^{3}$ \\ Qing Mei, ${ }^{3}$ Wenjuan Tang, ${ }^{3}$ \\ Yinghua Li, ' Hongsheng Liu, ${ }^{6}$ \\ Yongjian Zhou, ${ }^{3}$ Bing Zhu (D) \\ 'Central Laboratory, Guangzhou Institute of \\ Pediatrics, Guangzhou Women and Children's Medical \\ Center, Guangzhou Medical University, Guangzhou \\ 510120, People's Republic of China; ${ }^{2}$ State Key \\ Laboratory of Respiratory Disease, National Clinical \\ Research Center for Respiratory Disease, Guangzhou \\ Institute of Respiratory Health, First Affiliated Hospital \\ of Guangzhou Medical University, Guangzhou Medical \\ University, Guangzhou 510230, People's Republic of \\ China; ${ }^{3}$ Department of Gastroenterology and \\ Hepatology, Guangzhou Digestive Disease Center, \\ Guangzhou First People's Hospital, School of Medicine, \\ South China University of Technology, Guangzhou \\ 510180 , People's Republic of China; ${ }^{4}$ Department of \\ Orthopedics, Shenzhen Hospital (Futian) of \\ Guangzhou University of Chinese Medicine, Shenzhen \\ 518048, People's Republic of China; ${ }^{5}$ Shenzhen \\ Research Institute of Guangzhou University of Chinese \\ Medicine, Shenzhen 518048, People's Republic of \\ China; ${ }^{6}$ Department of Radiology, Guangzhou Women \\ and Children's Medical Center, Guangzhou Medical \\ University, Guangzhou 510120, People's Republic of \\ China
}

*These authors contributed equally to this work

Correspondence: Bing Zhu

Central Laboratory, Guangzhou Institute of Pediatrics, Guangzhou Women and Children's Medical Center, Guangzhou Medical University, Guangzhou 510I20, People's Republic of China

Tel +86 208I322725

Email zhubing2017@hotmail.com

Yongjian Zhou

Department of Gastroenterology and Hepatology, Guangzhou Digestive Disease Center, Guangzhou First People's

Hospital, School of Medicine, South China University of Technology, Guangzhou

510180 , People's Republic of China

Tel +86 2081045106

Email eyzhouyongjian@scut.edu.cn
Background: Delivery of therapeutic small interfering RNA (siRNA) via functionalized nanoparticles holds great promise for cancer therapy. However, developing a safe and efficient delivery carrier of siRNA is a challenging issue.

Methods: RGDfC peptide was used to modify the surface of selenium nanoparticles (SeNPs) to synthesize a biocompatible siRNA delivery vehicle (R-SeNPs), and MEF2DsiRNA was loaded onto R-SeNPs to prepare a functionalized selenium nanoparticle R-Se@MEF2D-siRNA. The chemical properties of R-SeNPs were characterized, and the anticancer efficacy as well as related mechanisms of R-Se@MEF2D-siRNA were further explored.

Results: R-Se@MEF2D-siRNA was significantly taken up by SKOV3 cells and could enter SKOV3 cells mainly in the clathrin-associated endocytosis way. The result of in vitro siRNA release demonstrated that R-Se@MEF2D-siRNA could release MEF2D-siRNA quicker in a microenvironment simulating a lysosomal environment in tumor cells compared to a normal physiological environment. The results of qRT-PCR assay proved that R-Se@MEF2D-siRNA could effectively silence the expression of the MEF2D gene in SKOV3 cells. R-Se@MEF2D-siRNA remarkably suppressed the proliferation of SKOV3 cells and further triggered its apoptosis. In addition, R-Se@MEF2D-siRNA had the capability to disrupt mitochondrial membrane potential (MMP) in SKOV3 cells and resulted in the overproduction of reactive oxygen species (ROS), indicating that mitochondrial dysfunction and ROS generation played an important role in the apoptosis of SKOV3 cells induced by R-Se@MEF2D-siRNA. In vivo, R-Se@MEF2D-siRNA also exhibited excellent antitumor activity mainly through decreasing tumor cells proliferation and triggering their apoptosis in tumor-bearing nude mice.

Conclusion: R-Se@MEF2D-siRNA provides an alternative strategy for ovarian cancer treatment in the clinic.

Keywords: ovarian cancer, siRNA delivery, gene therapy, tumor targeting, MEF2D

\section{Introduction}

Ovarian cancer is one of the deadliest malignancies in women because of its high recurrence rate, and the 5-year survival rate is less than $50 \%{ }^{1}$ Chemotherapeutic drugs are commonly used for ovarian cancer therapy. ${ }^{2,3}$ Nevertheless, most cancers including ovarian cancer have developed inherent or acquired resistance. ${ }^{4}$ Thus, some novel therapeutic strategy should be developed to improve the treatment outcomes. ${ }^{5}$ Short interfering RNA (siRNA) technology is one of the most effective options for cancer therapy. ${ }^{6}$ Viruses were usually applied to deliver siRNA in the previous studies. ${ }^{7}$ Nevertheless, viral carriers had the risk of immunogenicity and 
insertional mutagenesis. ${ }^{8,9}$ Thus, non-viral vehicle holds great potential for the gene treatment of cancers owing to its better safety. ${ }^{10}$

Selenium nanoparticles (SeNPs), due to their biocompatibility, easy surface modification and low toxicity, have attracted much attention in the design of drug/gene vehicles. ${ }^{11-13}$ On the one hand, the trace element selenium (Se) plays a crucial role for preventing cancer. ${ }^{14}$ On the other hand, the presence of Se in the human body can ensure proper function of the immune system. ${ }^{15}$ The advantage of SeNPs makes it superior to organic nanoparticles or inorganic metal nanoparticles to deliver drug/gene. ${ }^{16}$ Therefore, SeNPs have gradually developed one of the most promising chemotherapeutics gene/drugs vehicles. ${ }^{17}$ However, the lack of tumortargeted ability is the biggest obstacle to the application of nanoparticles as gene/drugs carriers. ${ }^{18-20}$ Thus, in this study, the positively charged peptide RGDfC was used to install on the surface of SeNPs to fabricate a tumor-targeted siRNA delivery vehicle (R-SeNPs). RGDfC has the ability to selectively bind with its receptor $\alpha v \beta 3$ integrin that is highly expressed in various kinds of cancer cells, including SKVO3 human ovarian cancer cells. ${ }^{21}$ In addition, R-SeNPs with a positive charge are prone to load with negative charge nucleic acid molecule siRNA via the electrostatic interaction. ${ }^{22}$

The myocyte enhancer factor 2 (MEF2) family, containing the MEF2-A, -B, -C, and -D subtypes, belongs to one of the human transcription factors. MEF2 family members play an important role in the occurrence and development of cancers. ${ }^{23}$ Previous research showed that expression of MEF2D was elevated in ovarian cancer, pancreatic cancer, non-small cell lung cancer, colorectal cancer, and so on. ${ }^{24}$ Thus, MEF2D-siRNA was loaded onto the surface of R-SeNPs to fabricate R-Se@MEF2D-siRNA, aiming at silencing MEF2D gene for ovarian cancer therapy. The anticancer efficacy and mechanisms of R-Se@MEF2D-siRNA were studied using ovarian cancer SKVO3 cells and a nude mice model.

\section{Materials and Methods Materials}

Lysotracker red, sodium selenite $\left(\mathrm{Na}_{2} \mathrm{SeO}_{3}\right)$, Hoechst 33342 , propidium (PI), and ascorbic acid ( $\mathrm{Vc}$ ) were obtained from Sigma (MO, USA). Annexin V/PI was purchased from Beyotime. Fetal bovine serum (FBS) was obtained from Gibco. All antibodies were purchased from CST (MA, USA). The sequence of MEF2D-siRNA was as follows: GCAACAGCCTAAACAAGGT.

\section{Fabrication and Characterizations of R-SeNPs}

Selenium nanoparticles (SeNPs) were synthesized in according with previous reported literature. ${ }^{10}$ Briefly, $0.5 \mathrm{~mL} \mathrm{Na}_{2}$ $\mathrm{SeO}_{3}(5 \mathrm{mM})$ and $0.5 \mathrm{~mL}$ vitamin $\mathrm{C}(20 \mathrm{mM})$ were mixed and the mixture solutions were gently stirred for 2 hours to prepare SeNPs. Then, $1 \mathrm{~mL}$ of RGDfC solution $(1 \mathrm{mg} / \mathrm{mL})$ was dripped to previous mixture solutions. The mixture solutions were stirred at $25^{\circ} \mathrm{C}$ for 3 hours to fabricate R-SeNPs. The pure R-SeNPs solution was acquired by dialyzing (MW cutoff $=3.5 \mathrm{kDa}$ ). The amount of RGDfC in nanoparticles was determined by high performance liquid chromatography (HPLC). Size distribution and zeta potential of R-SeNPs was examined by a Malvern Zetasizer. ${ }^{25}$ The polydispersity index of nanoparticles in this paper was in the range of $0.1 \sim 0.25$. Elemental composition analysis of R-SeNPs was carried out using energy dispersive X-Ray (EDX). The chemical structures of R-SeNPs were analyzed by Fourier transform infrared spectroscopy (FTIR). Morphology of R-SeNPs was visualized by transmission electron microscopy (TEM) ${ }^{26}$ Briefly, TEM samples were prepared by dripping the nanoparticles solution onto a holey carbon film on copper grids, and air dried for examination using TEM.

R-Se@MEF2D-siRNA was obtained via dripping MEF2D-siRNA $(100 \mathrm{nM})$ to R-SeNPs solution at $15^{\circ} \mathrm{C}$ for 35 minutes. The N/P (nitrogen atom/phosphorus atom) ratios of R-SeNPs/MEF2D-siRNA are 1:1, 2:1, 4:1, and 8:1. The concentrations of loaded MEF2D-siRNA were tested using a nanodrop 2000 spectrophotometer, as in previous literature. ${ }^{5}$

\section{Gel Electrophoresis Assay}

A gel retardation assay was performed by incubating R-SeNPs with MEF2D-siRNA for 25 minutes at various N/P rates to determine complete complex formation. R-Se@MEF2DsiRNA was transferred to an agarose gel electrophoresis (1\%) at $110 \mathrm{mV}$ for 20 minutes and then the gel imagings were visualized by a gel documentation system.

\section{Cell Culture}

HUVEC and SKOV3 cells were purchased from ATCC (Manassas, VA, USA) and incubated in Dulbecco's modified Eagle's medium (DMEM) containing 10\% FBS at $37^{\circ} \mathrm{C}$ with $5 \% \mathrm{CO}_{2}$.

\section{Cellular Uptake of R-Se@MEF2D-siRNA} SKOV 3 cells at the density of $5 \times 10^{4}$ cells $/ \mathrm{mL}$ were cultured in 6-well plate for 24 hours. Afterwards, SKOV3 cells were 
exposed to FAM-labeled R-Se@MEF2D-siRNA ${ }^{\text {FAM }}$ (100 $\mathrm{nM}$ of siRNA $\left.{ }^{\mathrm{FAM}}\right)$. The SKOV3 cells were washed, supplemented with fresh complete medium, and visualized under a fluorescence microscope. Uptake of R-Se@MEF2DsiRNA $^{\text {FAM }}$ in HUVEC was carried out by using the same method as above. The $\alpha_{v} \beta_{3}$ integrin expression in SKOV3 cells and HUVEC cells was analyzed by Western blot assay, as in a previous publication. ${ }^{10}$ In order to further verify whether RGDfC-mediated uptake occurred in SKOV3 cells, a competitive inhibition experiment has been performed. In brief, SKOV3 cells were pretreated with free RGDfC $(0.5 \mu \mathrm{g} / \mathrm{mL})$ for 2 hours. Subsequently, the cells were washed with cold PBS and exposed to R-Se@MEF2DsiRNA $^{\text {FAM }}$ for 1 hour, 2 hours, and 4 hours The uptake mechanisms of FAM-labeled R-Se@MEF2D-siRNA ${ }^{\text {FAM }}$ in SKOV3 cells were studied using various endocytosis inhibitors and analyzed by flow cytometer. ${ }^{27}$

\section{The Release of MEF2D-siRNA from R-SeNPs}

For assessing the release profiles of MEF2D-siRNA from R-SeNPs, 1 mg of R-Se@MEF2D-siRNA complexes with a N/P rate (8:1) were incubated with $1 \mathrm{~mL}$ PBS (pH 5.4 or 7.4) and transferred into a dialysis tube. The tubes were immersed in $100 \mathrm{~mL}$ of PBS (pH 5.4 or 7.4) under horizontal shaking (150 rpm). The released MEF2D-siRNA was detected for 15 hours by a spectromax quickdrop. At predetermined time points, aliquots of $0.5 \mathrm{~mL}$ outside the dialysis tubes were withdrawn for test and replenished with the equivalent volume of fresh PBS. The released amount of payload MEF2D-siRNA was determined by comparison with an experimentally determined standard curve. $^{28}$

\section{RT-qPCR Analysis}

SKOV3 cells in six-well plates were incubated for 24 hours to reach about $75 \%$ confluence. SKOV3 cells were co-incubated with $100 \mathrm{nM}$ equivalent siRNA of R-Se@MEF2D-siRNA or R-Se@siNC for 24 hours. The cells were rinsed and incubated in the fresh complete medium for another 24 hours. The cells without treatment were set as the control group. Total RNA was extracted from SKVO3 cells by TRIzol reagent in accordance with manufacturer's instruction. The RNA concentration was measured using a NanoDrop ${ }^{\mathrm{TM}} 1000$ Spectrophotometer. ${ }^{29}$ The $2^{-\Delta \Delta \mathrm{CT}}$ method was used to analyze the data. The primers sequences are shown in Table 1.
Table I The Primer Sequences of MEF2D and GAPDH for Quantitative Real-Time PCR

\begin{tabular}{|l|l|l|}
\hline Gene & Direction & Primers (5'-3') \\
\hline MEF2D & F & AGGGAAATAACCAAAAAACTACCAAA \\
& R & GCTACATGAACACAAAAACAGAGACC \\
\hline GAPDH & F & ATCCCATCACCATCTTCCAG \\
& R & ATGAGTCCTTCCACGATACC \\
\hline
\end{tabular}

\section{MTT Assay}

The cytotoxicity of R-Se@MEF2D-siRNA was detected using MTT assay. ${ }^{30}$ In brief, SKOV3 cells in a 96-well plate were cultured for 12 hours to acquire about $50 \%$ confluence. Then SKOV3 cells were exposed to R-Se@MEF2D-siRNA and R-Se@siNC (various siRNA equivalent concentrations) for 48 hours. The previous media were taken out and the fresh media containing MTT $(5 \mu \mathrm{g} / \mathrm{mL})$ were supplemented in each well. The 96-well plate was kept in an incubator at $37^{\circ} \mathrm{C}$ for another 4 hours. Then the media were taken out, and $150 \mu \mathrm{L}$ of DMSO was added to each well for shaking for 15 minutes. Finally, the cells were tested under a microplate reader at $570 \mathrm{~nm}^{31}$

\section{Flow Cytometry Assay}

Flow cytometry was used to analyze the cell cycle distribution and apoptosis. In brief, SKOV3 cells were treated with R-Se@MEF2D-siRNA or R-Se@siNC (100 nM of equivalent siRNA) for 24 hours, and then rinsed with PBS. The collected cells were fixed with methanol overnight and then stained with $1 \mu \mathrm{g} / \mathrm{mL}$ of propidium (PI) for 20 minutes before testing using flow cytometry. ${ }^{32}$

\section{Mitochondrial Membrane Potential $(\Delta \psi \mathrm{m})$ Assay}

For mitochondrial membrane potential (MMP) examination, the SKOV3 cells were treated with R-Se@MEF2DsiRNA or R-Se@siNC (100 nM of equivalent siRNA) for 24 hours and then were co-incubated with $1 \mu \mathrm{g} / \mathrm{mL}$ of JC1 for 15 minutes. Finally, the cells were washed and tested via a FACS flow cytometer. ${ }^{33}$

\section{Detection of Reactive Oxygen Species (ROS)}

ROS level of SKOV3 cells was examined as reported. ${ }^{34}$ Briefly, SKOV3 cells were treated with R-Se@MEF2DsiRNA or R-Se@siNC (100 nM of equivalent siRNA) for 24 hours before staining with $10 \mu \mathrm{M}$ DCFH-DA for 20 
minutes. Then the imaging of cells was captured under a fluorescence microscope.

\section{Xenograft Mouse Model}

All animal experiments were approved by the Ethics Committee of Guangzhou Medical University and performed according to the protocols and guidelines of the Experimental Animal Center of Guangzhou Medical University. A BALB/c nude mice model (6-week old) was used to assess the antitumor activity of R-Se@MEF2D-siRNA. Briefly, the tumor-bearing mice were prepared by subcutaneously injecting $5 \times 10^{6}$ SKOV3 cells in the abdomen. The tumor-bearing mice were intravenously injected with saline, R-Se@siNC, or R-Se@MEF2D-siRNA $(0.2 \mathrm{mg} / \mathrm{kg}$ of siRNA) once every 3 days after tumor volumes reached $100 \mathrm{~mm}^{3}$. Tumor volumes $\left(\mathrm{mm}^{3}\right)$ were calculated using the following formula: $\left(\right.$ length $\times$ width $\left.^{2}\right) / 2$.

The sections were stained with H\&E, Ki67, pp53, caspase 3 and TUNEL, and photographed under a microscope (Leica DMi8) for histologic analysis.

\section{Statistical Analysis}

Data are presented as the means \pm SD. A one-way ANOVA test was used to determine significance among groups. ${ }^{*} P<0.05$ and $* * P<0.01$ were considered significant and highly significant, respectively.

\section{Results and Discussion Characterization of R-SeNPs}

The morphology and size of the prepared selenium nanoparticles R-SeNPs were visualized by transmission electron microscopy (TEM), which indicated the uniform spherical particles of R-SeNPs $(\sim 80 \mathrm{~nm})$ (Figure 1A). Elemental compositions of R-SeNPs was analyzed by EDX. From Figure 1B, oxygen and carbon atom signal derived from RGDfC and a typical Se atom signal derived from SeNPs were detected in the spectrum of R-SeNPs, indicating the successful loading of RGDfC onto SeNPs. Moreover, FTIR spectrum of R-SeNPs (Figure 1C) demonstrated the similar FTIR spectrum of RGDfC with the characteristic peak at 1,658 and $1,533 \mathrm{~cm}^{-1}$ of amide derived from RGDfC, indicating the effectual preparation of R-SeNPs. The schematic illustration of the formation of RGDfC-Se@MEF2DsiRNA was presented in Scheme 1. From Figure 2, size distributions of R-SeNPs in deionized water and PBS observed for 15 days, and the result showed that R-SeNPs could keep a stable size of particles $(<150 \mathrm{~nm})$ for within 15 days. The favorable stability of R-SeNPs in water and PBS supports its biological application. From Figure 3A, zeta potential of SeNPs transformed from $-30.7 \mathrm{mV}$ to $13.7 \mathrm{mV}$ by loading with RGDfC, and the zeta potential of R-Se@MEF2D-siRNA was about 7.3 mV.

\section{Study on siRNA Loading Ability}

An agarose gel assay was applied to assess siRNA loading ability of R-SeNPs. Seen from Figure 3B, R-SeNPs could significantly bind MEF2D-siRNA and MEF2D-siRNA migration started to be retarded at a N/P ratio of 1:1. When a N/P ratio of R-SeNPs/MEF2D-siRNA reached 8:1, R-SeNPs could completely retard the migration of MEF2DsiRNA. These results showed that R-SeNPs had the ability to bind MEF2D-siRNA and protect its degradation.

\section{Selective Uptakes of R-Se@MEF2D- siRNA}

High uptake of therapeutic siRNA makes a great contribution to good treatment efficacy. Many studies showed that $\alpha_{v} \beta_{3}$ integrin, a receptor of RGD, was overexpressed in most cancer cells, including SKOV3 cells. ${ }^{35}$ The expression level of the integrin receptor on the cell membrane should be examined first in order to analyze whether the receptor contributes to the cellular uptake of R-Se@MEF2D-siRNA. The results indicated that the expression level of integrin receptor was higher in SKOV3 cells compared to HUVEC cells (Supplementary Figure S1), suggesting that RGDfC had a superior guided selectivity for SKOV3 cells to HUVEC cells. From Figure 4, the selective uptake of R-Se@MEF2DsiRNA in two cells was visualized under a fluorescence microscope, and the results showed that R-Se@MEF2DsiRNA exhibited greater cellular uptake in SKOV3 cells than that in HUVEC, suggesting RGDfC-modified functionalized selenium nanoparticles could enhance the uptake of MEF2D-siRNA in SKOV3 ovarian cancer cells. In order to further verify whether RGDfC-mediated uptake occurred in SKOV3 cells, a competitive inhibition experiment was carried out. Free RGDfC was added to SKOV3 cells first to block the interaction between RGDfC and its receptor integrin, and then R-Se@MEF2D-siRNA was added to the medium to study whether less R-Se@MEF2D-siRNA were taken by SKOV3 cells. As shown in Supplementary Figure S2, the pretreatment with free RGDfC in SKOV3 cells resulted in less uptake of R-Se@MEF2D-siRNA compared to SKOV3 cells without pretreatment, suggesting RGDfC-mediated targeting played an important role in the uptake of 


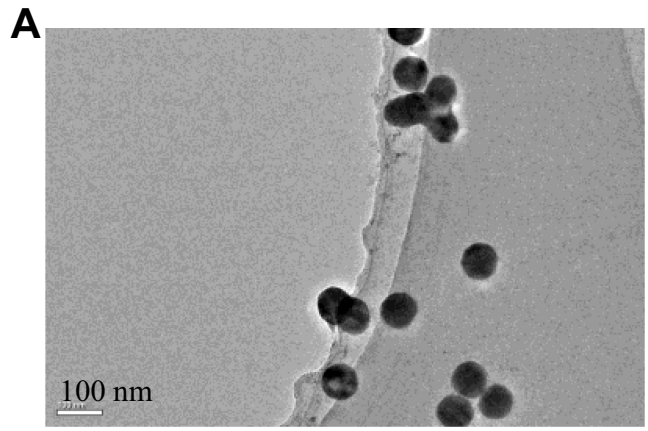

C
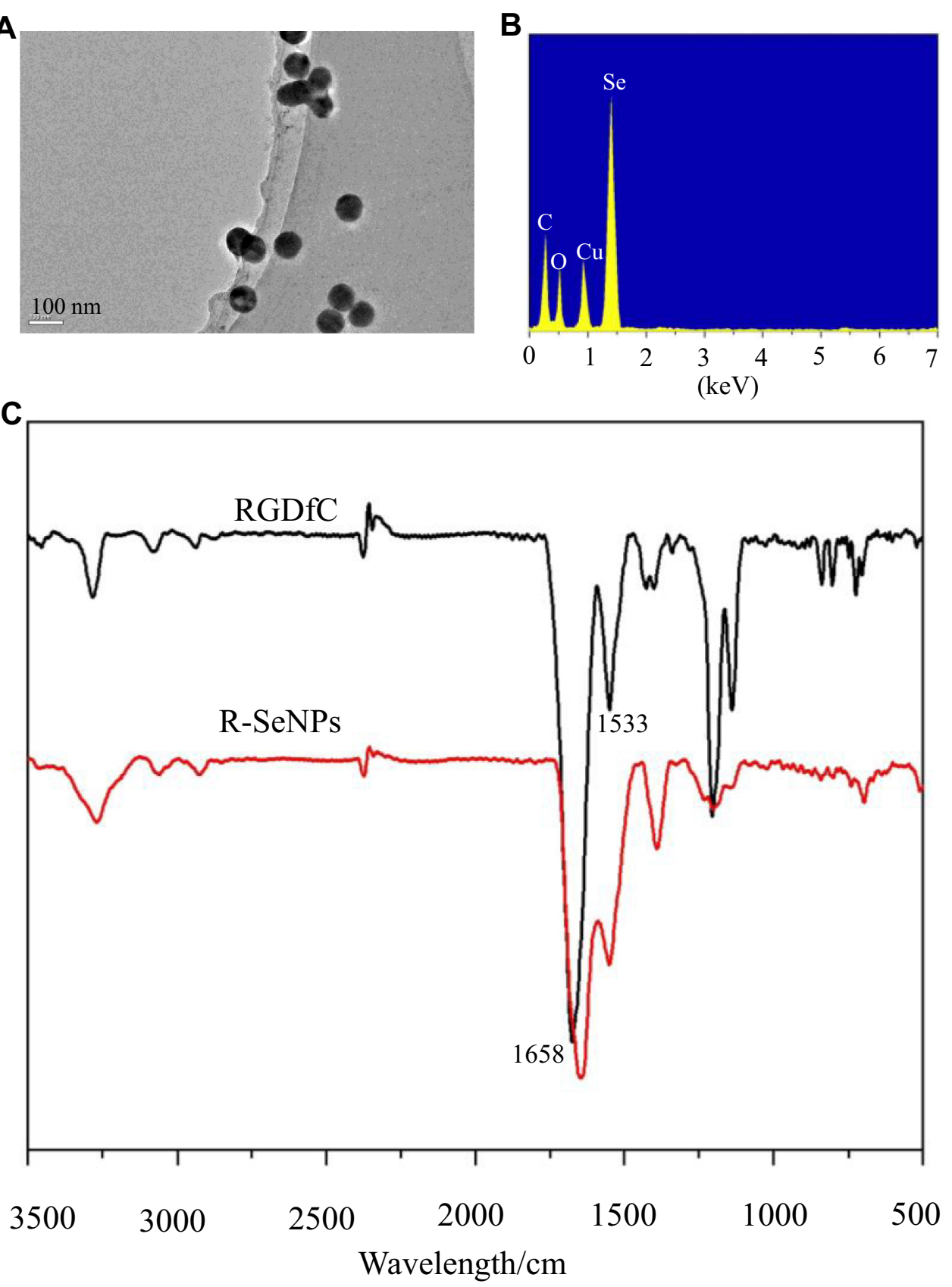

.
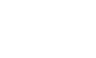


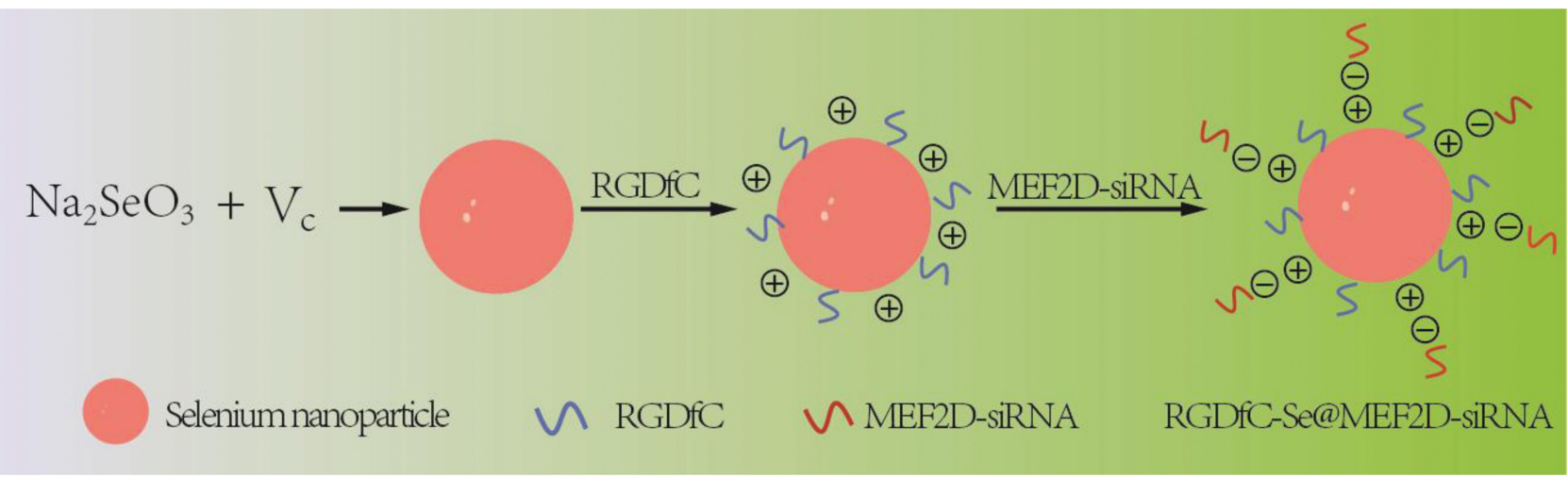

Scheme I Schematic illustration of the formation of RGDFC-Se@MEF2D-siRNA.

Abbreviations: RGDfC, Arg-Gly-Asp-D-Phe-Cys peptide; RGDfC-Se@MEF2D-siRNA, RGDfC-modified selenium nanoparticles loaded with MEF2D-siRNA; Vc, ascorbic acid.
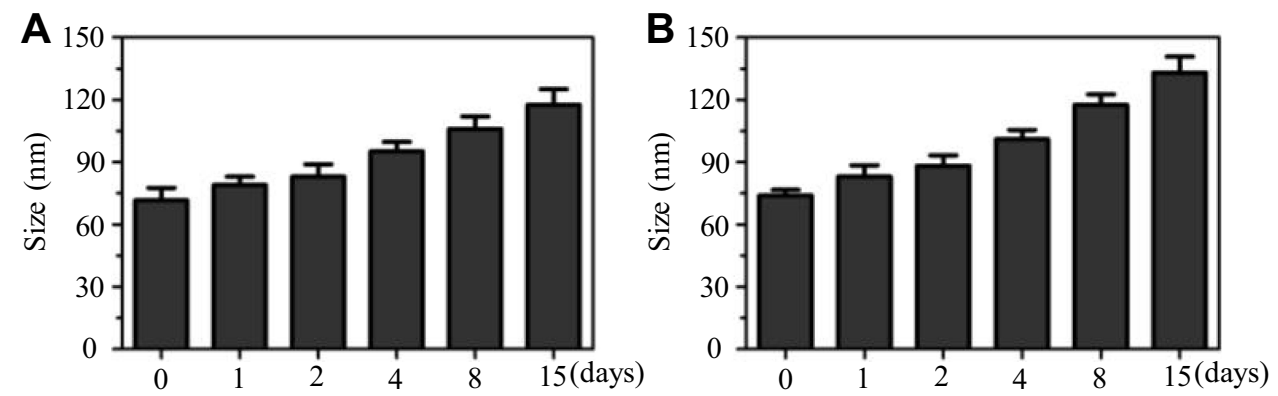

Figure 2 Particle size survey of R-SeNPs in deionized water (A) and PBS (B).

Abbreviations: PBS, phosphate buffer saline; R-SeNPs, RGDfC-modified selenium nanoparticles.
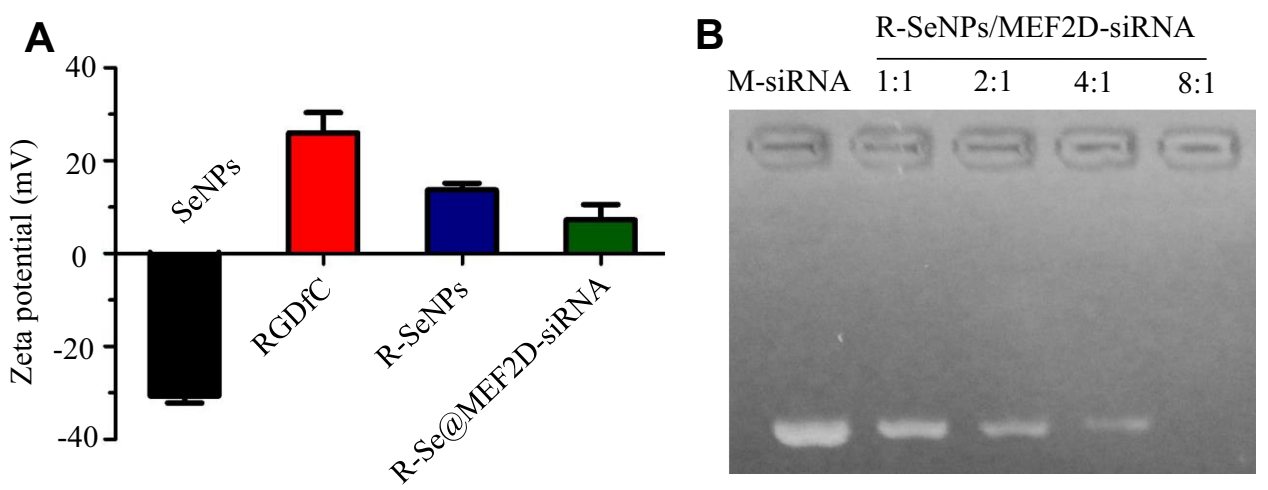

Figure 3 (A) Zeta potentials examination of SeNPs, RGDfC, R-SeNPs, and R-Se@MEF2D-siRNA. (B) MEF2D-siRNA stability in R-Se@MEF2D-siRNA at various N/P ratios was assessed by agarose gel electrophoresis assay.

Abbreviations: M-siRNA, MEF2D-siRNA; MEF2D-siRNA, MEF2D-targeted siRNA; R-SeNPs, RGDfC-modified selenium nanoparticles; R-Se@MEF2D-siRNA, RGDfCmodified selenium nanoparticles loaded with MEF2D-siRNA; SeNPs, selenium nanoparticles.

(macropinocytosis), and nystatin (caveolae-mediated endocytosis) were used to study endocytosis mechanisms of R-Se@MEF2D-siRNA. From Figure 5A, uptake of R-Se@MEF2D-siRNA by SKOV3 cells was decreased by $24 \%$ or $27 \%$ in the presence of nystatin or amiloride. Nevertheless, chlorpromazine-treatment made a great effect on the uptake of R-Se@MEF2D-siRNA, and the uptake was decreased by $48 \%$, suggesting that SKOV3 cells take up the R-Se@MEF2D-siRNA mainly by the clathrin-associated endocytosis pathway.

\section{The $\mathrm{pH}$-Sensitive Release of MEF2D-siRNA}

The releases of MEF2D-siRNA from R-SeNPs were detected in PBS of pH 5.4 and pH 7.4 simulating an acidic endosomes/ 

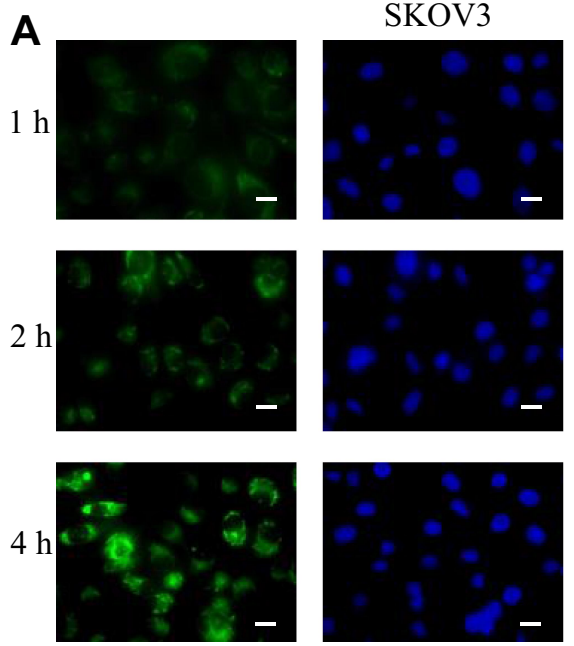

R-Se@MEF2D-siRNA

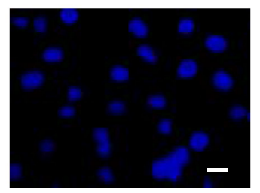

Hoechst 33342
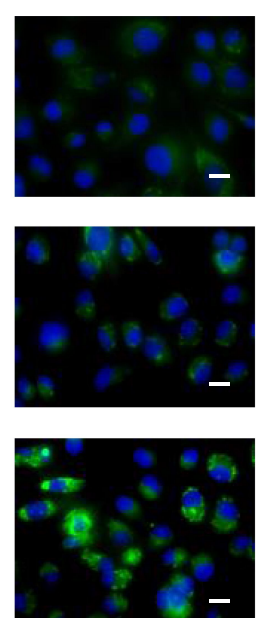

Merged
B
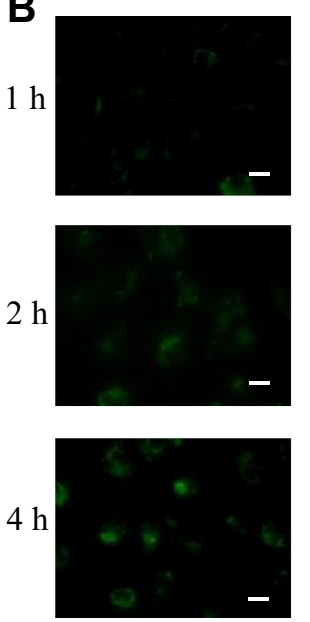

R-Se@MEF2D-siRNA
HUVEC
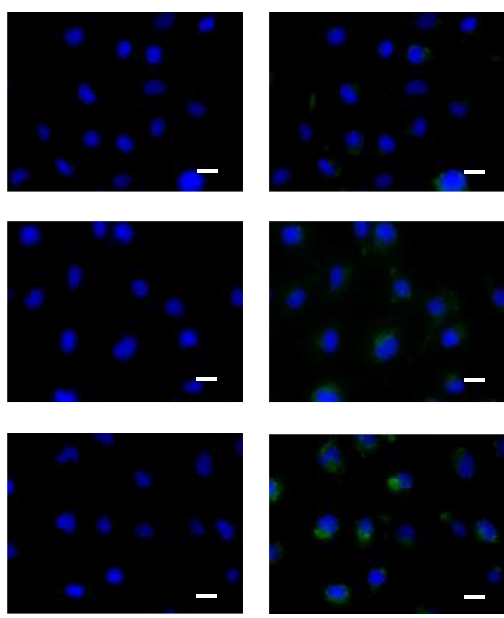

Hoechst 33342
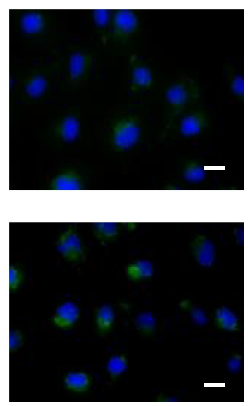

Merged

Figure 4 The uptake of R-Se@MEF2D-siRNA was visualized by fluorescence microscope. (A) R-Se@MEF2D-siRNA was taken up by SKOV3 cells. (B) R-Se@MEF2DsiRNA was taken up by HUVEC. Scale bar indicates $20 \mu \mathrm{m}$.

Abbreviations: Hoechst 33342, Bisbenzimide H33342; HUVEC, human umbilical vein endothelial cells; R-Se@MEF2D-siRNA, RGDfC-modified selenium nanoparticles loaded with MEF2D-siRNA; SKOV3, human ovarian cancer cells.

\section{A}

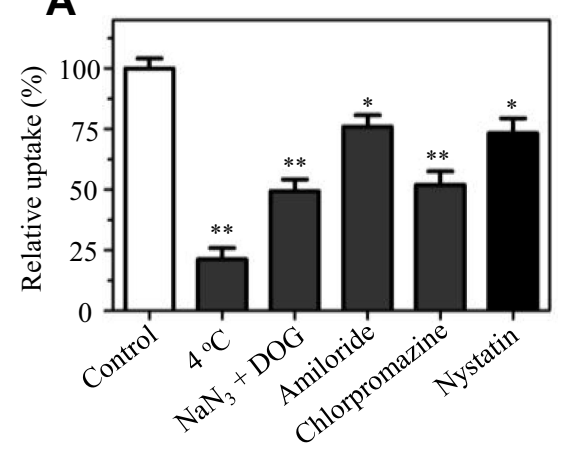

B

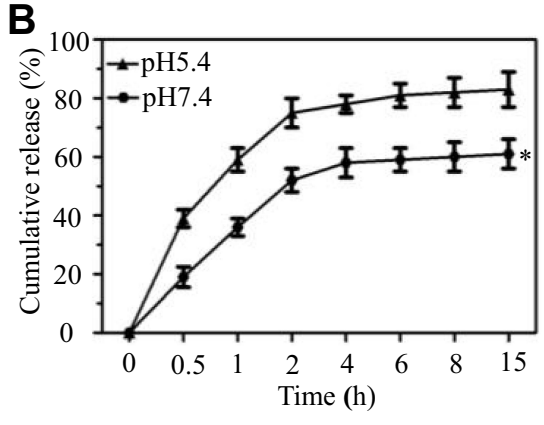

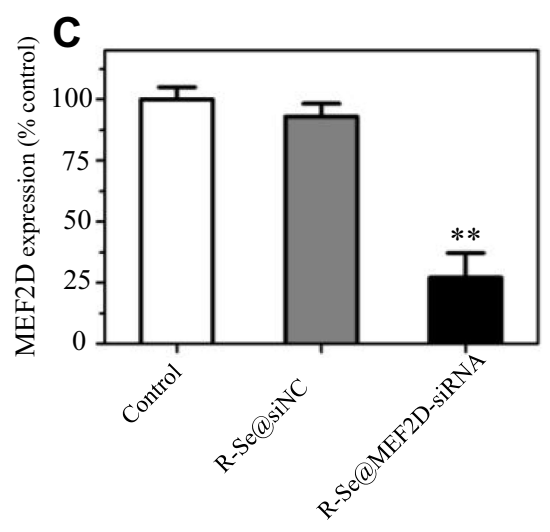

Figure 5 (A) The uptake of R-Se@MEF2D-siRNA in SKOV3 cells was influenced by low temperature and endocytosis inhibitors. $* P<0.05$, $* * P<0.01$ vs control. (B) The release of MEF2D-siRNA from R-SeNPs at pH5.4 and pH7.4. $* P<0.05$ vs pH5.4 group. (C) Relative expressions of MEF2D in SKOV3 cells were examined using qRT-PCR. $* * P<0.01$ vs control group.

Abbreviations: DOG, 2-deoxy-D-glucose; R-Se@MEF2D-siRNA, RGDfC-modified selenium nanoparticles loaded with MEF2D-siRNA; R-Se@siNC, RGDfC-modified selenium nanoparticles loaded with siRNA (negative control).

lysosomes microenvironment and normal physiological environment, respectively. ${ }^{37}$ From Figure $5 \mathrm{~B}$, the obvious burst releases of MEF2D-siRNA in both $\mathrm{pH}$ values were observed within the initial 2 hours. Furthermore, R-Se@MEF2DsiRNA in PBS of pH 5.4 exhibited quicker release of MEF2DsiRNA and the release rate was about $83 \%$ at 15 hours. Nevertheless, the release rate of MEF2D-siRNA in $\mathrm{pH} 7.4$ $(61 \%)$ was lower than that in $\mathrm{pH} 5.4(83 \%)$ at 15 hours. This phenomenon could explain that acidic conditions increased the protonation of R-SeNPs, and thus weakened electrostatic interactions between R-SeNPs and MEF2D-siRNA, which promoted the release of MEF2D-siRNA from R-SeNPs. The acid-sensitive siRNA release feature of R-Se@MEF2DsiRNA exhibited unique advantages in cancer therapy.

\section{R-Se@MEF2D-siRNA Silences the Expression of the MEF2D Gene}

R-SeNPs was applied to deliver MEF2D-siRNA to SKOV3 cells, aiming at silencing the gene expression of MEF2D. A quantitative real time polymerase chain reaction (qRTPCR) was applied to assess the mRNA level of MEF2D in SKOV3 cells. Seen from Figure 5C, R-Se@MEF2D-siRNA remarkably decreased the MEF2D mRNA level of SKOV3 cells. However, R-Se@siNC hardly influenced the 
expression of the MEF2D gene. The above results revealed that R-Se@MEF2D-siRNA could significantly silence the MEF2D gene in SKOV3 cells.

\section{R-Se@MEF2D-siRNA Inhibits the Proliferation of SKOV3 Cells}

The proliferation inhibition of SKOV3 cells was assessed using MTT assay. From Figure 6A, the proliferation of SKOV3 cells was remarkably suppressed by R-Se@MEF2D-siRNA and the cell viability rate was $39.6 \%$ after 48 hours. Nevertheless, no obvious effect on viability of cells treated with R-Se@siNC was observed, indicating that R-Se@MEF2D-siRNA inhibits the proliferation of SKOV3 ovarian cancer cells by silencing gene expression of MEF2D. The carrier itself (R-Se@siNC) did not exhibit obvious cytotoxicity against SKOV3 cells at such low concentrations as used in this study. Besides, R-Se@MEF2D-siRNA exhibited weak cellular uptake in HUVEC and had slight cytotoxicity against HUVEC (Figure 6B), further confirming the good biocompatibility of R-Se@MEF2D-siRNA. This finding was consistent with previous publications. ${ }^{13,29}$
Apoptosis of SKOV3 cells induced by R-Se@MEF2DsiRNA was assessed using flow cytometry. From Figure 6C, a sub-G1 apoptosis peak in the R-Se@MEF2D-siRNA group was more obvious $(20.15 \%)$ compared to the R-Se@siNC group (6.42\%) or untreated control group $(6.19 \%)$, proving that R-Se@MEF2D-siRNA obviously induced the apoptosis of SKOV3 cells. In addition, R-Se@MEF2D-siRNA did not have a significant effect on cell cycle distribution of SKOV3 cells.

\section{R-Se@MEF2D-siRNA Induces Mitochondria Dysfunction and ROS Overproduction}

The loss of mitochondrial membrane potential (MMP, $\Delta \Psi \mathrm{m}$ ) can initiate apoptosis of tumor cells. Thus, we used flow cytometry to examine whether R-Se@MEF2D-siRNA triggered SKOV3 cells apoptosis by damaging the MMP. ${ }^{38}$ The change of $\Delta \Psi \mathrm{m}$ of the cells were detected by staining with JC-1 to study the initiation of cell apoptosis, in which the red fluorescence indicates normal cells and the green fluorescence signal indicates apoptotic cells with mitochondrial dysfunction. Seen from Figure 7A, a part of red fluorescence
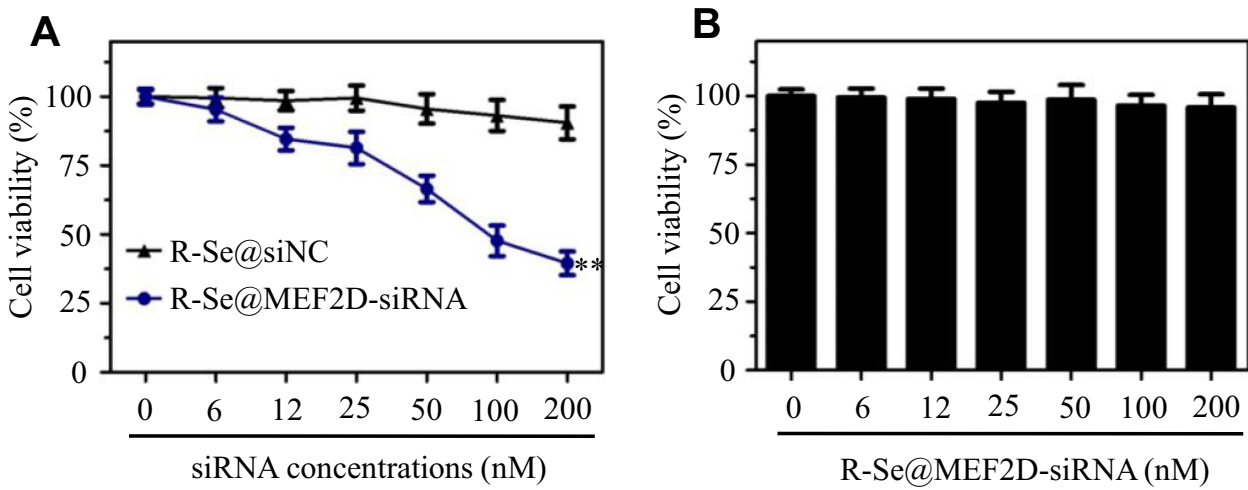

C

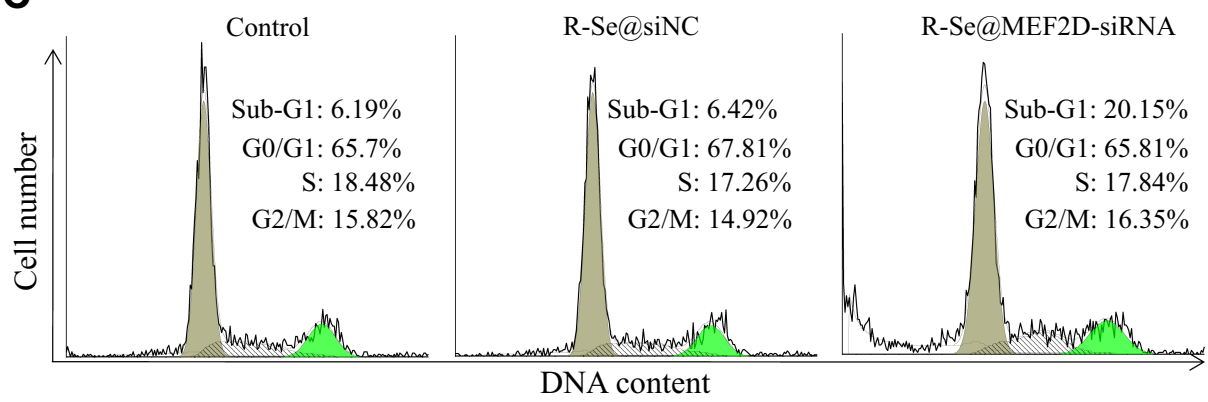

Figure 6 (A) Cytotoxicity of SKOV3 cells exposed to various concentrations of R-Se@siNC and R-Se@MEF2D-siRNA after 48 hours. (B) Cytotoxicity of HUVEC exposed to various concentrations of R-Se@MEF2D-siRNA.**P<0.0I vs R-Se@siNC. (C) Cell cycle phase distribution of nuclear DNA in R-Se@siNC or R-Se@MEF2D-siRNAtreated SKOV3 cells was determined by flow cytometry.

Abbreviations: R-Se@MEF2D-siRNA, RGDfC-modified selenium nanoparticles loaded with MEF2D-siRNA; R-Se@siNC, RGDfC-modified selenium nanoparticles loaded with siRNA (negative control). 
transformed into green fluorescence, indicating R-Se@MEF2D-siRNA elevated the mitochondria depolarization of SKOV3 cells. The mitochondrial dysfunctions of SKOV3 cells exposed to R-Se@MEF2D-siRNA remarkably increased from $1.7 \%$ (untreated cells) to $21.32 \%$. The above result indicates that the mitochondrial dysfunction induced by R-Se@MEF2D-siRNA makes an important contribution to the apoptosis of SKOV3 ovarian cancer cells.

Overproduction of reactive oxygen species (ROS) caused by antitumor drugs plays an essential role in the apoptosis and death of cancer cells. ${ }^{39}$ Therefore, in this study, the ROS level of SKOV3 cells was detected via fluorescence method. Figure 7B shows that R-Se@MEF2D-siRNA-treatment resulted in an obvious increase of DCF green fluorescence signal in SKOV3 cells, suggesting the ROS overproduction. Nevertheless, R-Se@siNC did not affect the MMP and the ROS level in comparison with the control group. The above results indicate that mitochondrial dysfunction and ROS overproduction induced by R-Se@MEF2D-siRNA play an important role in apoptosis of SKOV3 cells.

\section{In vivo Anti-Tumor Efficacy and Toxicity \\ Studies}

The SKOV3 tumor xenograft was applied to assess the in vivo anticancer efficacy of R-Se@MEF2D-siRNA. From Figure 8A, as expected, R-Se@MEF2D-siRNA significantly delayed the tumor growth compared to the saline group (control) and R-Se@siNC group (negative control), verifying good antitumor efficacy of R-Se@MEF2DsiRNA. As seen from Figure 8B, the body weights of mice during the treatment period showed a slight increase, indicating that R-Se@MEF2D-siRNA was biocompatible materials in mice at the used dose. The anticancer mechanism of R-Se@MEF2D-siRNA was further elaborated by histological studies. Ki67-staining was used to examine the proliferation of tumor cells. The caspase-3, pp53, and TUNEL staining were applied to study tumor cells
A

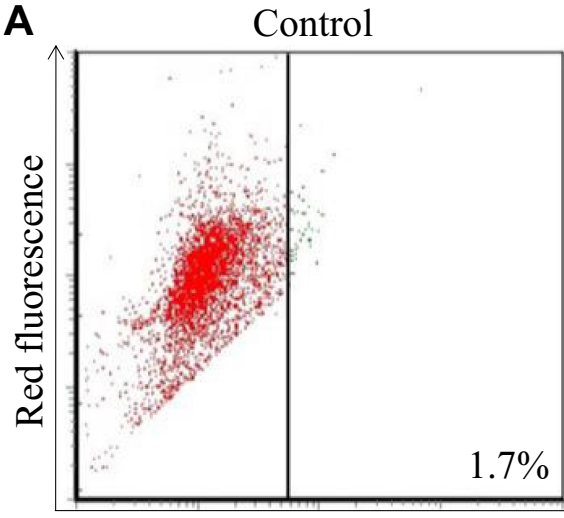

B

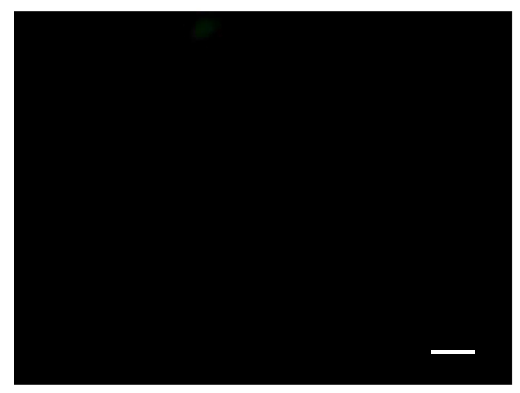

Control
R-Se@siNC

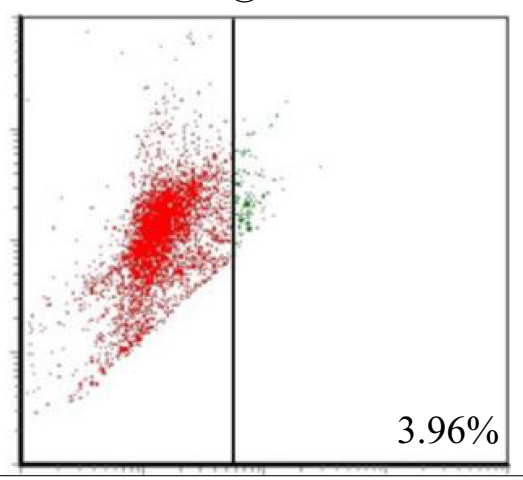

Green fluorescence

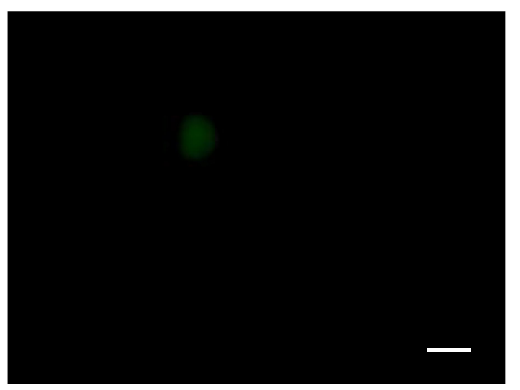

R-Se@siNC

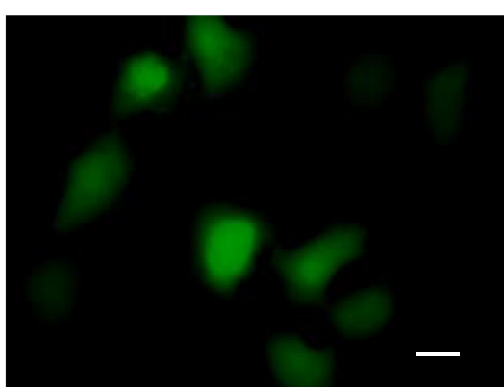

R-Se@MEF2D-siRNA

Figure 7 (A) R-Se@MEF2D-siRNA induced changes of MMP in SKOV3 cells. The MMP values of treated cells were examined by flow cytometry using JC-I staining. (B) R-Se@MEF2D-siRNA increased the ROS level of SKOV3 cells. The treated SKOV3 cells were co-incubated with DCFDA for 30 minutes and then visualized by a fluorescence microscope to measure intracellular ROS levels. Scale bar indicates $20 \mu \mathrm{m}$.

Abbreviations: R-Se@MEF2D-siRNA, RGDfC-modified selenium nanoparticles loaded with MEF2D-siRNA; R-Se@siNC, RGDfC-modified selenium nanoparticles loaded with siRNA (negative control). 
apoptosis (Figure 8C). Compared to the saline-treatment group, the treatment of R-Se@MEF2D-siRNA observably reduced the Ki67-positive tumor cells, suggesting that the proliferation of tumor cells was suppressed by R-Se@MEF2D-siRNA. As expected, R-Se@MEF2DsiRNA could obviously induce tumor cell apoptosis. These results indicated that R-Se@MEF2D-siRNA presented a significant antitumor efficacy in ovarian cancer treatments by suppressing the proliferation of SKOV3 ovarian cancer cells and inducing its apoptosis.

To assess in vivo toxicity of R-Se@MEF2D-siRNA, heart, kidneys, liver, lung and spleen of mice were collected and stained with H\&E. As shown in Figure 9, the H\&E stained heart, kidneys, liver, lung, and spleen tissue sections in the R-Se@MEF2D-siRNA-treatment group showed no obvious abnormality compared to saline or R-Se@siNC negative groups, further proving the good in vivo biocompatibility of R-Se@MEF2D-siRNA. Thus, R-Se@MEF2D-siRNA exhibited promising potential to be a prodrug for ovarian cancer therapy.

\section{Conclusions}

In this study, a tumor-targeted siRNA delivery carrier R-SeNPs was fabricated to deliver MEF2D-siRNA to SKOV3 cells for ovarian cancer therapy. R-Se@MEF2DsiRNA exhibited significant uptake in SKOV3 cells by clathrin-associated endocytosis and release of MEF2D-siRNA from R-SeNPs in acidic condition was quicker than that in normal physiological condition. R-Se@MEF2D-siRNA could efficaciously silence gene expression of MEF2D in SKOV3 cells and suppress proliferations of SKOV3 cells, and trigger SKOV3 cells apoptosis. More importantly, R-Se@MEF2D-siRNA exerted significant antitumor activity with low toxic side-effects in tumor-bearing nude mice.
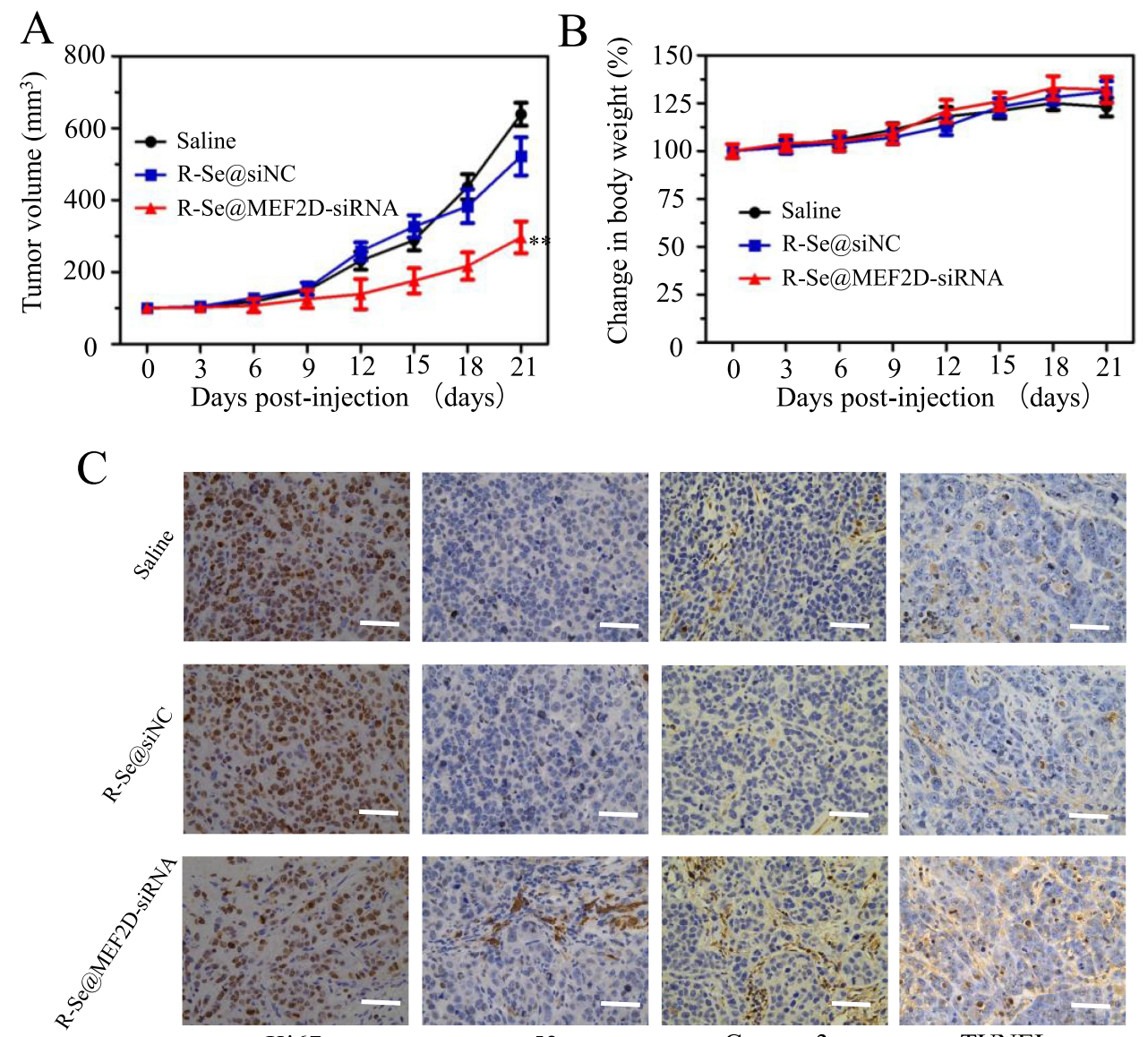

pp53

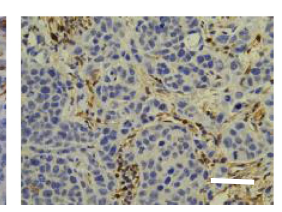

Caspase 3

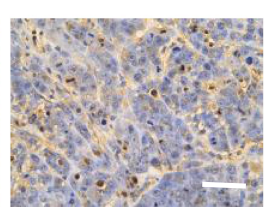

TUNEL

Figure 8 (A) Tumor volume change curves in different treated groups during 21 day treatment. $* * P<0.01$ vs saline. (B) The weight of mice was recorded during 21 day treatment. (C) The immunohistochemical analysis of tumor tissues in different treated mice. Scale bar indicates $50 \mu \mathrm{m}$.

Abbreviations: R-Se@MEF2D-siRNA, RGDfC-modified selenium nanoparticles loaded with MEF2D-siRNA; R-Se@siNC, RGDfC-modified selenium nanoparticles loaded with siRNA (negative control). 

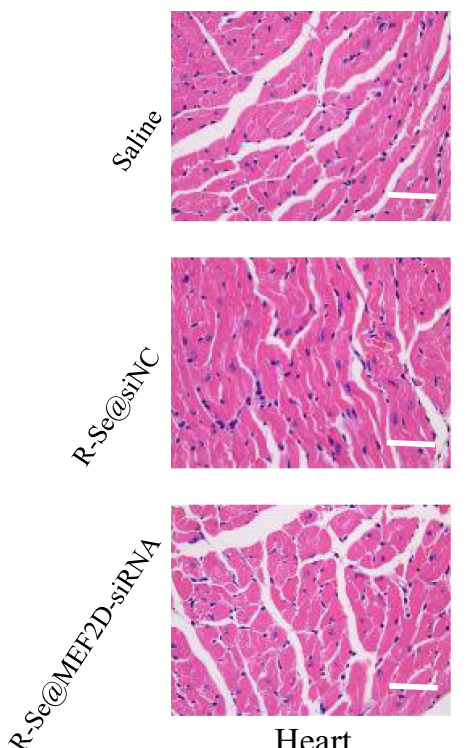

Heart
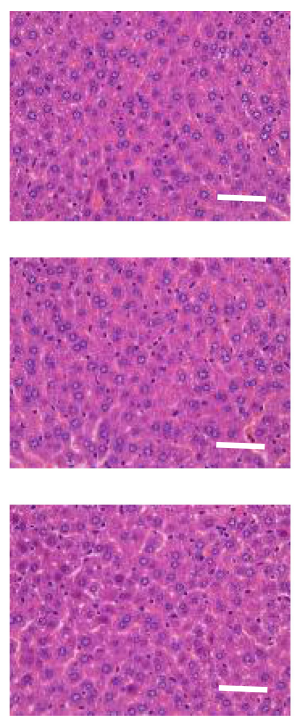

Liver
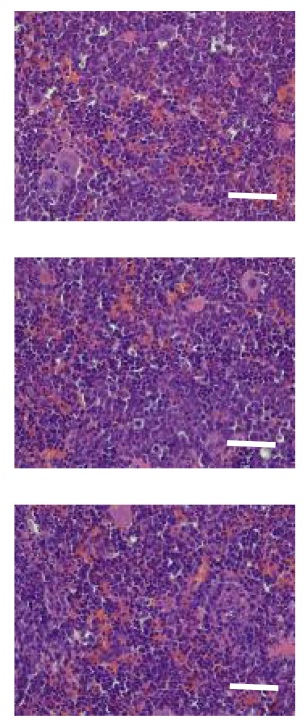

Spleen
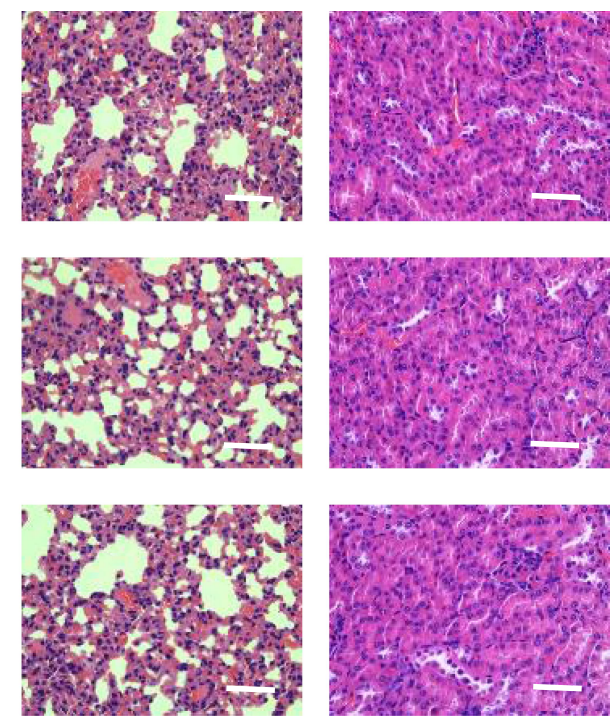

Lung

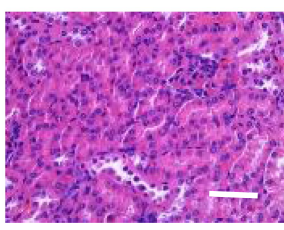

Kidney

Figure 9 H\&E stained heart, kidneys, liver, lung, and spleen tissues from mice treated with saline, R-Se@MEF2D-siRNA or R-Se@siNC. Bar indicates 50 $\mu$ m. Abbreviations: R-Se@MEF2D-siRNA, RGDfC-modified selenium nanoparticles loaded with MEF2D-siRNA; R-Se@siNC, RGDfC-modified selenium nanoparticles loaded with siRNA (negative control).

Taken together, R-Se@MEF2D-siRNA holds promising potential for ovarian cancer gene therapy.

\section{Acknowledgments}

This work was supported by the Science and Technology Planning Project of Guangdong Province (2014A020212024, 2015A020211002), the National Natural Science Foundation of China (81970507, 82004395), the Natural Science Foundation of Guangdong Province (2020A1515010100), the Technology Planning Project of Guangzhou City (2019 04010132; 201804010142), Innovative Clinical Technique of Guangzhou (2019GX05), China Postdoctoral Science Foundation (2018M633088), Scientific Research Project of Guangdong Provincial Bureau of Traditional Chinese Medicine (20192054).

\section{Disclosure}

The authors declare no conflicts of interest.

\section{References}

1. Zhang Q, Kuang G, He S, et al. Photoactivatable prodrug-backboned polymeric nanoparticles for efficient light-controlled gene delivery and synergistic treatment of platinum-resistant ovarian cancer. Nano Lett. 2020;20:3039-3049. doi:10.1021/acs.nanolett.9b04981

2. Karimi S, Fouani MH, Moshaii A, Nikkhah M, Hosseinkhani S, Sheikhnejad R. Development of dual functional nucleic acid delivery nanosystem for DNA induced silencing of $\mathrm{Bcl}-2$ oncogene. Int J Nanomedicine. 2020;15:1693-1708. doi:10.2147/IJN.S236217
3. Paro AD, Hossain M, Webster TJ, Su M. Monte Carlo and analytic simulations in nanoparticle-enhanced radiation therapy. Int J Nanomedicine. 2016;11:4735-4741. doi:10.2147/IJN.S114025

4. Mahmoodi Chalbatani G, Dana H, Gharagouzloo E, et al. Small interfering RNAs (siRNAs) in cancer therapy: a nano-based approach. Int J Nanomedicine. 2019;14:3111-3128. doi:10.2147/IJN.S200253

5. Zhang $\mathrm{F}$, Zhao L, Wang $\mathrm{S}$, et al. Construction of a biomimetic magnetosome and its application as a SiRNA carrier for high-performance anticancer therapy. Adv Funct Mater. 2018;28:1703326. doi:10.1002/adfm.201703326

6. Guo Z, Li S, Liu Z, Xue W. Tumor-penetrating peptide-functionalized redox-responsive hyperbranched poly(amido amine) delivering siRNA for lung cancer therapy. ACS Biomater Sci Eng. 2018;4:988-996. doi:10.1021/acsbiomaterials.7b00971

7. Vaidya AM, Sun Z, Ayat N, et al. Systemic delivery of tumor-targeting siRNA nanoparticles against an oncogenic LncRNA facilitates effective triple-negative breast cancer therapy. Bioconjugate Chem. 2019;30:907-919. doi:10.1021/acs.bioconjchem.9b00028

8. Sakurai Y, Hada T, Kato A, Hagino Y, Mizumura W, Harashima H. Effective therapy using a liposomal siRNA that targets the tumor vasculature in a model murine breast cancer with lung metastasis. Mol Ther Oncolytics. 2018;11:102-108. doi:10.1016/j.omto.2018.10.004

9. Rehman M, Ihsan A, Madni A, et al. Solid lipid nanoparticles for thermoresponsive targeting: evidence from spectrophotometry, electrochemical, and cytotoxicity studies. Int $J$ Nanomedicine. 2017;12:8325-8336. doi:10.2147/IJN.S147506

10. Xia Y, Tang G, Guo M, et al. Silencing KLK12 expression via RGDfC-decorated selenium nanoparticles for the treatment of colorectal cancer in vitro and in vivo. Mater Sci Eng C-Mater. 2020;110:110594. doi:10.1016/j.msec.2019.110594

11. Guo M, Li Y, Lin Z, et al. Surface decoration of selenium nanoparticles with curcumin induced HepG2 cell apoptosis through ROS mediated p53 and AKT signaling pathways. RSC Adv. 2017;7 (83):52456-52464. doi:10.1039/C7RA08796A

12. Xia Y, Xu T, Zhao M, et al. Delivery of doxorubicin for human cervical carcinoma targeting therapy by folic acid-modified selenium nanoparticles. Int $J$ Mol Sci. 2018;19:3582. doi:10.3390/ ijms 19113582 
13. Li Y, Lin $\mathrm{Z}$, Gong $\mathrm{G}$, et al. Inhibition of H1N1 influenza virus-induced apoptosis by selenium nanoparticles functionalized with arbidol through ROS-mediated signaling pathways. $J$ Mater Chem B. 2019;7(27):4252-4262. doi:10.1039/C9TB00531E

14. Xia Y, Xu T, Wang $\mathrm{C}$, et al. Novel functionalized nanoparticles for tumor-targeting co-delivery of doxorubicin and siRNA to enhance cancer therapy. Int J Nanomedicine. 2017;13:143-159. doi:10.2147/ IJN.S148960

15. Lin $\mathrm{Z}, \mathrm{Li} \mathrm{Y}, \mathrm{Xu} \mathrm{T}$, et al. Inhibition of Enterovirus 71 by selenium nanoparticles loaded with siRNA through bax signaling pathways. $A C S$ Omega. 2020;5(21):12495-12500. doi:10.1021/acsomega.0c01382

16. Gong G, Li Y, He K, et al. The inhibition of H1N1 influenza induced apoptosis by sodium selenite through ROS-mediated signaling pathways. $R S C A d v$. 2020;10:8002-8007. doi:10.1039/C9RA09524A

17. Xia Y, Tang G, Chen Y, et al. Tumor-targeted delivery of siRNA to silence Sox2 gene expression enhances therapeutic response in hepatocellular carcinoma. Bioact Mater. 2021;6:1330-1340. doi:10.1016/ j.bioactmat.2020.10.019

18. Yang X, Zhang C, Li A, Wang J, Cai X. Red fluorescent $\mathrm{ZnO}$ nanoparticle grafted with polyglycerol and conjugated RGD peptide as drug delivery vehicles for efficient target cancer therapy. Mater Sci Eng C-Mater. 2019;95:104-113. doi:10.1016/j.msec.2018.10.066

19. Zhu P, Gao S, Lin H, et al. Inorganic nanoshell-stabilized liquid metal for targeted photonanomedicine in NIR-II biowindow. Nano Lett. 2019;19(3):2128-2137. doi:10.1021/acs.nanolett.9b00364

20. Tao S-C, Guo S-C, Zhang C-Q. Modularized extracellular vesicles: the dawn of prospective personalized and precision medicine. $A d v$ Sci. 2018;5:1700449. doi:10.1002/advs.201700449

21. Sun L, Zheng C, Webster TJ. Self-assembled peptide nanomaterials for biomedical applications: promises and pitfalls. Int J Nanomedicine. 2017;12:73-86. doi:10.2147/IJN.S117501

22. Xia Y, Lin Z, Li Y, et al. Targeted delivery of siRNA using RGDfC-conjugated functionalized selenium nanoparticles for anticancer therapy. J Mater Chem B. 2017;5:6941-6952. doi:10.1039/ C7TB01315A

23. Chow R, Wessels JM, Foster WG. Brain-derived neurotrophic factor (BDNF) expression and function in the mammalian reproductive Tract. Hum Reprod Update. 2020;26:545-564. doi:10.1093/humupd/dmaa008

24. Xiang J, Sun H, Su L, et al. Myocyte enhancer factor 2D promotes colorectal cancer angiogenesis downstream of hypoxia-inducible factor 1alpha. Cancer Lett. 2017;400:117-126. doi:10.1016/j. canlet.2017.04.037

25. Li K, Lu X, Zhang Y, Liu K, Huang Y, Liu H. Bi3TaO7/Ti3C2 heterojunctions for enhanced photocatalytic removal of water-borne contaminants. Environ Res. 2020;185:109409. doi:10.1016/j. envres.2020.109409

26. Huang Y, Long B, Tang M, et al. Bifunctional catalytic material: an ultrastable and high-performance surface defect $\mathrm{CeO} 2$ nanosheets for formaldehyde thermal oxidation and photocatalytic oxidation. Appl Catal B Environ. 2016;181:779-787. doi:10.1016/j.apcatb.2015. 08.047

27. Xia Y, Zhong J, Zhao M, et al. Galactose-modified selenium nanoparticles for targeted delivery of doxorubicin to hepatocellular carcinoma. Drug Deliv. 2019;26:1-11. doi:10.1080/ 10717544.2018.1556359

International Journal of Nanomedicine

\section{Publish your work in this journal}

The International Journal of Nanomedicine is an international, peerreviewed journal focusing on the application of nanotechnology in diagnostics, therapeutics, and drug delivery systems throughout the biomedical field. This journal is indexed on PubMed Central, MedLine, CAS, SciSearch ${ }^{\circledR}$, Current Contents ${ }^{\mathbb{B}} /$ Clinical Medicine,
28. Zhou Y, Yu Q, Qin X, et al. Improving the anticancer efficacy of laminin receptor-specific therapeutic ruthenium nanoparticles (RuBB-Loaded EGCG-RuNPs) via ROS-dependent apoptosis in SMMC-7721 cells. ACS Appl Mater Interfaces. 2015;8:15000-15012. doi:10.1021/acsami.5b02261

29. Xia Y, Tang G, Wang C, et al. Functionalized selenium nanoparticles for targeted siRNA delivery silence Derlin1 and promote antitumor efficacy against cervical cancer. Drug Deliv. 2019;27:15-25. doi:10.1080/10717544.2019.1667452

30. Yin T, Yang L, Liu Y, Zhou X, Sun J, Liu J. Sialic acid (SA)-modified selenium nanoparticles coated with a high blood-brain barrier permeability peptide-B6 peptide for potential use in Alzheimer's disease. Acta Biomater. 2015;25:172-183. doi:10.1016/j.actbio.2015.06.035

31. Li Y, Xia Y, Liu K, et al. Constructing Fe-MOF-derived Z-scheme photocatalysts with enhanced charge transport: nanointerface and carbon sheath synergistic effect. ACS Appl Mater Interfaces. 2020;12:25494-25502. doi:10.1021/acsami.0c06601

32. Zheng W, Yin T, Chen Q, et al. Co-delivery of Se nanoparticles and pooled SiRNAs for overcoming drug resistance mediated by P-glycoprotein and class III beta-tubulin in drug-resistant breast cancers. Acta Biomater. 2016;31:197-210. doi:10.1016/j.actbio.2015.11.041

33. Xia Y, Xiao M, Zhao M, et al. Doxorubicin-loaded functionalized selenium nanoparticles for enhanced antitumor efficacy in cervical carcinoma therapy. Mater Sci Eng C. 2020;106:110100. doi:10.1016/ j.msec. 2019.110100

34. Gurunathan S, Qasim M, Park CH, et al. Cytotoxicity and transcriptomic analyses of biogenic palladium nanoparticles in human ovarian cancer cells (SKOV3). Nanomaterials. 2019;9(5):787. doi:10.3390/ nano 9050787

35. Xia Y, Guo M, Xu T, et al. siRNA-loaded selenium nanoparticle modified with hyaluronic acid for enhanced hepatocellular carcinoma therapy. Int J Nanomedicine. 2018;13:1539-1552. doi:10.2147/IJN.S157519

36. Zeng P, Xu Y, Zeng C, Ren H, Peng M. Chitosan-modified poly(D, L-lactide-co-glycolide) nanospheres for plasmid DNA delivery and HBV gene-silencing. Int J Pharm. 2011;415:259-266. doi:10.1016/j. ijpharm.2011.05.053

37. Xia Y, Chen Y, Hua L, et al. Functionalized selenium nanoparticles for targeted delivery of doxorubicin to improve non-small-cell lung cancer therapy. Int $J$ Nanomedicine. 2018;13:6929-6939. doi:10.2147/IJN.S174909

38. Zhang J, Wang Q, Guo Z, et al. High-fidelity trapping of spatialtemporal mitochondria with rational design of aggregation-induced emission probes. Adv Funct Mater. 2019;29(16):1808153. doi:10.1002/adfm.201808153

39. Gupta PK, Pappuru S, Gupta S, Patra B, Chakraborty D, Verma RS. Self-assembled dual-drug loaded core-shell nanoparticles based on metal-free fully alternating polyester for cancer theranostics. Mater Sci Eng C-Mater. 2019;101:448-463. doi:10.1016/j.msec.2019.03.041

Journal Citation Reports/Science Edition, EMBase, Scopus and the Elsevier Bibliographic databases. The manuscript management system is completely online and includes a very quick and fair peer-review system, which is all easy to use. Visit http://www.dovepress.com/ testimonials.php to read real quotes from published authors. 